\title{
tic\&société
}

Vol. 9, $\mathbf{N}^{\circ}$ 1-2 | 1 er semestre 2015 - 2ème semestre 2015

Les religions au temps du numérique

\section{De la mise en scène pudique comme expression d'une morale musulmane sur Youtube}

\section{Lucie LE GUEN-FORMENTI}

\section{(2) OpenEdition \\ Journals}

Édition électronique

URL : http://journals.openedition.org/ticetsociete/1824

DOI : 10.4000/ticetsociete. 1824

Éditeur

Association ARTIC

Référence électronique

Lucie LE GUEN-FORMENTI, « De la mise en scène pudique comme expression d'une morale

musulmane sur Youtube », tic\&société [En ligne], Vol. 9, № 1-2| 1er semestre 2015 - 2ème semestre 2015, mis en ligne le 01 février 2016, consulté le 19 avril 2019. URL : http://journals.openedition.org/ ticetsociete/1824; DOI : 10.4000/ticetsociete.1824 
tic\&société - 9 (1-2), 2015

De la mise en scène pudique comme expression d'une morale musulmane sur Youtube

Lucie LE GUEN-FORMENTI

le-guen-lucie@hotmail.fr 


\title{
Lucie LE GUEN-FORMENTI
}

\section{De la mise en scène pudique comme expression d'une morale musulmane sur Youtube}

\begin{abstract}
Étudiante en master 2 Industries culturelles et créatives à I'Université Paris 8, Lucie Le Guen-Formenti a rédigé au cours de l'année 2014-2015 un mémoire de recherche en sciences de l'information et de la communication à l'Université Paris 3 intitulé Ambivalence et légitimations de nouvelles religiosités musulmanes : le cas du rap français. " $A$ ton avis, on va mourir pieux ? Allah u A3lim, c'qui est sûr, c'est qu'partis comme on est, on va pas mourir vieux " portant sur les légitimations individuelles de huit rappeurs français. Elle a effectué un stage de recherche pour le projet Minweb, «Minorités, identités numériques et circulation des messages politiques sur le web dans le Sahara " (Ceped, Paris Descartes, IRD) concernant les représentations des martyrs dans le cyber activisme des groupes indépendantistes au Nord du Mali. Elle est cofondatrice et coorganisatrice de l'Acemup (association pour un colloque étudiant sur les musiques populaires).
\end{abstract}

Résumé : Avec le développement d'internet apparaissent de nouvelles pratiques religieuses en ligne : l'adaptation d'une pratique religieuse musulmane à un milieu de vie profane entraîne l'émergence de rationalisations différentes via ce média. L'objet est ici d'interroger la production de vidéos déposées sur Youtube consacrées à la religion musulmane et de déterminer leur rapport à une valeur religieuse centrale : la pudeur. Nous espérons ainsi faire apparaître un mode de valorisation de soi, entre producteurs (Youtubeurs) et récepteurs (viewers), qui va placer la pudeur en élément central 
De la mise en scène pudique

comme expression d'une morale musulmane sur Youtube

de la justification éthique et morale de l'individu, tout en accompagnant un lien communautaire.

Mots-clés : Islam, Youtube, pudeur, honneur, communauté, vidéo.

Abstract: Depicting modesty as an expression of a muslim morality on Youtube. As the web expands, new religious practices are appearing online. New rationalizations emerge from the adaptation of Muslim believes to a profane life in the West. This article focuses on Youtube videos whose subject is Islam, and examines how they position themselves in relation to a major theological value: modesty. We hope to identify a starting point for self-valorization between the producers (Youtubers) and the audience (viewers) that situates modesty as a major element of ethical and personal morality, at the same time illustrating a communitarian link.

Keywords: Islam, Youtube, modesty, honor, community, video.

Resumen : Mostrar el pudor como expresión de una moralidad musulmana en Youtube. Con el desarrollo del internet aparecen nuevas prácticas religiosas en línea: la adaptación de una práctica religiosa musulmanas a un entorno de vida profana implica la emergencia de racionalizaciones diferentes. El presente texto es de analizar la producción de videos subidos a youtube relacionados con la religión musulmana e identificar su relación con un valor religioso central como es el pudor. Así, esperamos hacer de esta manera surge un modo de valorización personal entre productores (youtubers) y receptores (viewers) que va a instituir el pudor como elemento central de la justificación ética y moral del individuo, acompañando un vínculo comunitario.

Palabras clave : Islam, Youtube, pudor, honor, comunidad.

Si les évènements géopolitiques actuels permettent de mettre sur le devant de la scène un grand nombre de recherches relatives à l'exercice de la foi musulmane au $X X I^{e}$ siècle, le propos n'est pas ici de s'intéresser à l'islam politique, 


\section{Lucie LE GUEN-FORMENTI}

tel qui peut être perçu dans un monde qui fait suite au 11 septembre 2001, mais plutôt de voir la manière selon laquelle une identité musulmane va se construire et s'instituer auprès d'un public jeune et occidental. À travers l'analyse de chaînes Youtube anglophones, nous avons la possibilité de nous intéresser à un public jeune, tant au niveau des émetteurs que des récepteurs, et à une pratique abordant des problématiques localisées et circonstanciées, voire exclusives. Ces chaînes, d'origine anglaise, américaine, australienne ou canadienne, proposent un nouveau message religieux via un média particulier, selon un usage encore peu investi par des producteurs francophones.

\section{1. État de la recherche}

Dès lors, l'intérêt va être de comprendre comment la religion se trouve adaptée, tant au niveau de ses modalités que de son lien communautaire, et ce, via le prisme d'internet.

\subsection{Adapter l'islam}

\subsubsection{Mettre en ligne la religion}

Avec internet se développe un nouveau rapport à la foi : l'islam en ligne ne se définit plus seulement comme un système de lois (« sharia ») mais à partir d'un ensemble de valeurs à visée universaliste, correspondant à une nouvelle vision de la religiosité, non plus fondée sur la tradition religieuse mais sur le rapport personnel à celle-ci. Une modalité de socialisation (Roy, 2004) apparaît, via des modérateurs qui vont répéter et reformuler un modèle de conduite identifiable par leurs pairs. Le croyant ne cherche pas à réformer l'islam, mais à en tirer une expérience immédiate et interne, qui doit s'intégrer dans la société actuelle. Cette valorisation individuelle est accompagnée d'un mouvement de discrédit des institutions

tic\&société - 9 (1-2), 2015 
De la mise en scène pudique

comme expression d'une morale musulmane sur Youtube

traditionnelles, qui perdent du terrain sur l'enseignement, et ce, notamment sur internet: la religion se trouve ainsi "sécularisée " par des autodidactes se réappropriant un discours orthodoxe strict qui apporte des éléments de réponse à des champs délaissés par les institutions traditionnelles (mode, vie quotidienne, travail, etc.). C'est dans cette mesure qu'un discours salafi' peut plaire: la recherche d'une tradition normée et sanctifiée (soit la religion « telle qu'elle était exercée du temps du prophète $»)$, dégagée des problématiques actuelles et concrètes, peut ainsi s'adapter à toutes les sociétés. Ce phénomène se trouve favorisé par internet qui entraîne intrinsèquement une homogénéisation du discours et des ressources religieuses (Roy, 2004) : les sources doivent être peu nombreuses afin de faciliter l'acquisition personnelle de chacun, la langue (souvent anglaise) doit rester simple pour être comprise de tous. Or, la manière d'appréhender les outils proposés est quant à elle très traditionnaliste : comme dans les courants sunnites non salafi, on retrouve l'apprentissage du Coran par cœur, les jeux de questions-réponses pour découvrir le vrai, les discussions polémiques fondées sur des citations du Coran et des Hadiths. Se modifie également la

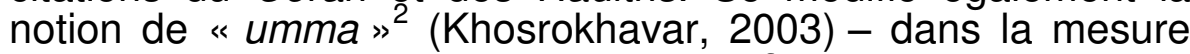
où le modèle qu'elle propose est confronté à de nouveaux modes de construction et de réussite sociale.

\subsubsection{Créer un lien communautaire signifiant}

Internet ne va pas permettre la création d'une communauté en tant que telle, mais plutôt d'enrichir un lien préexistant (Hugon, 2011). Ce lien est proposé comme l'expression d'un groupe recentré sur des valeurs (Hugon, 2011, cite Tahar Jabir Alalvani), un imaginaire commun, une temporalité particulière

\footnotetext{
${ }^{1}$ Le concept émique (i.e. propre aux acteurs) «Salafi », utilisé dans le sens littéral de la nisba arabe désigne ce qui appartient au mouvement salaf, soit ce qui se réfère aux proches du prophète. II est ici utilisé de préférence à la dénomination francophone.

${ }^{2}$ De «um», communauté fondée sur un ensemble de valeurs, et non sur un territoire comme dans le cas d'une nation (Dupret 2011).
} 


\section{Lucie LE GUEN-FORMENTI}

qui nécessitent l'implication et l'acceptation des membres. II n'exprime pas réellement une fin, mais plutôt un intérêt pour une expérience collective forte autour d'un imaginaire. Dans cette mesure, l'expérience communautaire laisse une grande place aux phénomènes d'individuation, en réaction à ces conceptions partagées. Le web, et en particulier les forums, produit un contexte d'usage de langue (religiosité, ethnicité, niveau social...) généralement spécifique au discours oral (Dupret, 2010). Un corpus moral ${ }^{3}$ et doctrinal ${ }^{4}$ est développé par la communauté, qui va le réinvestir dans son discours pour créer une intelligibilité propre et exclusive (Dupret, 2010 cite Jayyusi). Pourtant, une discontinuité réalité/web existe (Roy, 2005) : les sites, forums et blogs ne renvoient que rarement à des espaces hors ligne mais plutôt à la toile elle-même, créant ainsi un « espace de substitution ».

Face à ce nouveau type d'imaginaire (individualisation, conception du religieux en occident), l'islam se trouve en compétition avec d'autres religions comme sur un marché d'offre et de demande. La « néo-umma » (Roy, 2004) est donc le fruit de réalités plurielles, chaque individu effectuant des choix selon son rapport à sa terre d'origine, sa terre d'accueil, l'apparition de sous-cultures... produisant une communauté décentralisée, où l'individu tient une place importante et y recherche une solution aux sentiments de solitude et de vacuité.

\subsubsection{Développer un islam compétitif}

Cette idée d'une vision religieuse se rapprochant de l'idéologie marchande peut être attribuée à un "come-back pieux » des classes moyennes urbaines (Haenni, 2005). L'islam

\footnotetext{
${ }^{3}$ Comportant la générosité, la sincérité, la politesse (Hirschkind, 2013).

4 Utilisation du Coran, de formules de politesses traditionnelles pour marquer les débuts et fins de tours de paroles.
}

tic\&société - 9 (1-2), 2015 


\section{De la mise en scène pudique \\ comme expression d'une morale musulmane sur Youtube}

emprunte au marché ses catégories de pensée, l'idée de réalisation de soi. Dans un même temps, les phénomènes culturels perdent de leur " islamité » : l'apprentissage de l'arabe est en baisse et les codes iconographiques et médiatiques des médias occidentaux sont intégrés aux pratiques (Haenni, 2005 cite Gymnastiar et Khaled). Le but de la religion change également: il ne s'agit plus de convaincre d'une "vérité ultime " mais plutôt d'ajuster l'offre religieuse aux attentes réelles ou supposées du public cible. Pour cela, elle va proposer une démarche séduisante qui rompt avec les techniques traditionnelles tout en réinscrivant le message religieux dans un contexte global. L'offre religieuse va venir se « mouler » dans les attentes et représentations d'une majorité musulmane conservatrice ${ }^{5}$ qui propose une sécularisation des biens cultuels. Dès lors, internet se place comme un outil d'éducation du musulman afin de lui permettre de bien pratiquer les rituels et de constituer un rapport identitaire cohérent avec son environnement social (Cesari, 2004). Un « islam d'identité " dont la pratique en ligne va dégager l'apparition de nouvelles formes d' " interactions pieuses " (Hirschkind, 2012). Les pratiques de "mu'aththir "6 et de "da'wa»", qui permettent de faire le lien entre des éléments religieux et des éléments profanes, vont venir appuyer le lien communautaire. Des termes jusqu'alors relevant du sacré revêtent ainsi une signification plus large et personnelle (voire non religieuse), selon la sensibilité des individus. Se développe ainsi un espace d'échange entre les usagers, perçu comme «à part 》 de l'internet profane par les participants: dès lors que les commentaires des utilisateurs ne s'inscrivent plus dans une optique "mu'aththir », les usagers recherchant un espace pieux vont abandonner la page au profit d'une autre.

${ }^{5}$ Et non pas radicale, comme avancé par Olivier Roy (2004).

6 Peut être compris comme « influent». Nom arabe traduisant à la fois les termes touchant, émouvant, efficace, excitant, passionnant mais incluant également une idée de soulagement émotionnel.

7 Sentiment pieux. 


\section{Lucie LE GUEN-FORMENTI}

\subsection{Mise en scène de soi}

\subsubsection{La pudeur dans la religion musulmane}

Comme à la salle de prière, ce n'est pas réellement la foi de l'individu qui est valorisée, mais le caractère identifiable de sa piété. L'individuation du rapport à la foi va venir concurrencer celui présent dans la tradition musulmane, régi par une association de valeurs genrées, produits d'un contrôle social : le « namoûs », partiellement défini dans le Coran ${ }^{8}$.

Ce concept regroupe à la fois le cas féminin privé de la " h'aya ${ }^{9}$ et son pendant masculin public: l'honneur, « charaf» (Bouhdiba, 1984). Cette association de valeurs produit une perception du monde à la fois personnelle et collective, dans le sens où elle va organiser le comportement public des individus envers les autres et envers Dieu (via les intentions) en excluant de cette sphère tout ce qui n'y est pas strictement nécessaire. La pudeur induit également une spatialisation genrée (Ossman, 2002) effective (un seul sexe peut être présent sur place, à l'abri des regards du sexe opposé), à travers le développement d'une « seconde nature »

${ }^{8}$ Coran, 24 ; 30-31: «Dis aux croyants de baisser leurs regards et de garder leur chasteté. C'est plus pur pour eux. Allah est, certes, parfaitement connaisseur de ce qu'ils font. Et dis aux croyantes de baisser leurs regards, de garder leur chasteté, et de ne montrer de leurs atours que ce qui en paraît et qu'elles rabattent leur voile sur leurs poitrines; et qu'elles ne montrent leurs atours qu'à leurs maris, ou à leurs pères, ou aux pères de leurs maris, ou à leurs fils, ou aux fils de leurs maris, ou à leurs frères, ou aux fils de leurs frères, ou aux fils de leurs sœurs, ou aux femmes musulmanes, ou aux esclaves qu'elles possèdent, ou aux domestiques mâles impuissants, ou aux garçons impubères qui ignorent tout des parties cachées des femmes. Et qu'elles ne frappent pas avec leurs pieds de façon que l'on sache ce qu'elles cachent de leurs parures. Et repentez-vous tous devant Allah, Ô croyants, afin que vous récoltiez le succès". Dans la version arabe, il faut entendre " chasteté » au sens de furûjahûnna, soit « intrinsèquement lié à l'intimité sexuelle féminine ».

${ }^{9}$ Désigne la pudeur, la honte, la crainte du blâme.

tic\&société - 9 (1-2), 2015 


\section{De la mise en scène pudique \\ comme expression d'une morale musulmane sur Youtube}

permanente, délimitant la sphère publique de celle privée (Bouhdiba, 1984) mais aussi idéologique : c'est l'idée d'une séparation plus que son application réelle qui importe. Si elle est signifiée d'une quelconque manière, la pudeur est préservée. Néanmoins, la pudeur est également juge (Tillion, 1966). Elle va servir en cas de litige ou de questionnement à estimer la valeur religieuse et morale d'un individu. Dans cette mesure, la pudeur détient un rôle plus ambigu que celui seul de marqueur du permis et de l'interdit social.

\subsubsection{Extimité et narration de soi}

Si le comportement en public doit être dicté par un ensemble de règles écartant les données intimes, dès lors se pose la question de la mise en ligne de soi. En effet, la production de contenus numériques implique un "dévoilement de soi" (Denouël, 2011) dans une logique relationnelle : les contenus publiés (comme les vidéos Youtube ici étudiées) sont organisés de manière à être lus et commentés par un public plus ou moins identifié, qui va, d'une certaine manière, orienter le ton et le type de discours dans une recherche d'approbation, une "gestion d'impressions". Cette mise en récit du soi relève du concept d' " extimité ${ }^{10}$ (Tisseron, 2011), facilité par internet, qui, proposant un anonymat relatif, permet une certaine désinhibition ainsi qu'un oubli partiel du public (Denouël, 2011; Tisseron, 2011). Néanmoins, ce public contraint à un certain réalisme : un jeu de "clair-obscur » permettant d'adapter différentes facettes d'une même identité aux exigences d'un public hétérogène et à visées multiples, mais toujours sur un ton informel (Cardon, 2011).

Cette extimité permet la production de réputations par essence multiples, relatives, provisoires et contextuelles (Tisseron, 2011). Celles-ci ne relèvent pas de qualités intrinsèques, mais de qualités attribuées par les récepteurs et qui sont construites par des effets de style, un ton émotionnel,

\footnotetext{
10 Partage avec autrui d'éléments intimes visant à leur reconnaissance comme originaux.
} 


\section{Lucie LE GUEN-FORMENTI}

la visibilité et le style de personnalité employé (Tisseron cite Zafirau). C'est le jugement subjectif des individus qui va désormais classer et organiser les réputations, une «auteurisation de soi » (Cardon, 2013), où la production va être associée intimement à son émetteur. Le discours conversationnel, l'implication personnelle, et l'interpénétration avec la vie hors ligne sont autant de facteurs qui vont rattacher les productions aux personnes comme des signaux identitaires et qui vont apporter du crédit à l'énoncé. Le public participe également à la construction narrative dans le sens où le producteur va l'intégrer, par rapport de connivence à une narration personnelle dans laquelle il puisse se reconnaître (Dupont et Tillier, 2012).

\section{La construction du «je» public, entre pudeur et extimité}

Les concepts de pudeur et d'honneur subissent une certaine remise en cause avec la production en ligne: ces valeurs ne semblent plus effectives de par la publicisation d'éléments intimes et la visibilité qui leur est accordée. Néanmoins, la rigueur exigée pour la reconnaissance implique un comportement social particulier, ainsi qu'un respect de normes de publications. Ainsi, la pudeur semble être une codification dépassant les distinctions de genre, puisqu'elle va non seulement s'appliquer au cas féminin, comme dans la tradition musulmane, mais va aussi régir le comportement en ligne masculin, comme code moral dépendant de la question de l'honneur. La pudeur connaît une seconde adaptation transgenre, plus litterale : traditionnellement réservée à la gestion du privé et de l'interaction sociale, avec internet elle va également régir la face publique des individus et les modalités de jugements qui s'y appliquent, et ce de manière accentuée par rapport aux interactions directes. Une sorte de transfert de supports et d'applications, de modes de relations sociales et de genres de communications se forme ainsi. 
De la mise en scène pudique

comme expression d'une morale musulmane sur Youtube

Non seulement la pudeur est en grande partie composante de l'honneur de l'individu, mais elle va aussi participer à la définition de sa valeur; elle sera donc à la fois code moral et juge de ce même code. L'intérêt sera dès lors de voir la mesure selon laquelle cette évaluation de la valeur de l'individu semble être problématique sur Youtube.

L'intérêt sera porté à quatre chaînes de l'interface qu'est Youtube $^{11}$, et aux opportunités qu'elle offre à ses utilisateurs afin d'aménager la religion aux problématiques rencontrées, et ainsi produire un discours pertinent pour leurs pairs. Ces chaînes $^{12}$ ont été sélectionnées selon leur pertinence à une série de mots clefs ("fashion veil ", " muslim spoken word", «muslim short movie»). De plus (excepté celle d'Amenakin), ces vidéos ont été traduites par " Darifton et Compagnie », une chaîne qui relaie les vidéos d'origine anglophone pour un public francophone, attestant d'une certaine mise en valeur de ces productions.

- Celle d'Amenakin ${ }^{13}$, jeune anglaise d'origine indienne, à l'origine de la marque Pearl Daisy, et d'un grand nombre de vidéos de mode et de vie privée sur sa chaîne.

- Celle de Lebo2196, chaîne de Kamal Saleh, jeune australien publiant à l'origine fatwas et khutbas de sheikhs. Depuis deux ans il publie principalement des slams $s^{15}$ de son cru, en réponse à des discours tenus sur Youtube qu'il trouve offensants pour la religion ${ }^{16}$.

\footnotetext{
11 Les vidéos ont été visionnées entre novembre 2013 et mars 2014. L'analyse ici proposée correspond donc aux chaînes Youtube telles qu'elles apparaissaient à cette période, et ce, quelles que soient les mutations qu'elles aient connu depuis, notamment quant aux noms des producteurs et diffuseurs.

${ }^{12}$ Dénomination officielle usitée sur Youtube.

13 Depuis dénommée Amena.

${ }^{14}<$ https://www.youtube.com/channel/UC9SIIYeOQb2vrTQ3opSCXkg>, dernière consultation le 14 mars 2014.

${ }_{15}^{15}$ En anglais, "Muslim Spoken Word".

16 <https://www.youtube.com/channel/UCxBR44IXdJYOgsfHcIDMQuQ>, dernière consultation le 14 mars 2014.
} 


\section{Lucie LE GUEN-FORMENTI}

- Celle d'Islamicevent, une association musulmane à but apologétique. Les vidéos proposées sont parmi les plus consultées dans la catégorie «mini film musulman »"17.

- Celle de West Dawn Media, start-up canadienne de production numérique, dont les court-métrages jouent généralement sur des références filmiques et humoristiques. ${ }^{18}$

Une fois toutes les vidéos visionnées, c'est la question de la pudeur qui a été centrale : à l'aide d'un tableau les vidéos ont été comparées selon leur taux d'exposition (rapport au nombre de like/vues/commentaires) et se sont vues attribuer des mots clefs afin de déterminer les thématiques qui semblaient le plus intéresser les viewers ${ }^{19}$. Ainsi, les vidéos relatives à la pudeur (ou à l'honneur, la modestie, ou encore au couple ou l'amour, bien que relevant a priori de la sphère privée, et donc régies par l'idée de pudeur) semblaient être celles suscitant le plus de réactions de la part du public, mais également leur nombre était en augmentation à mesure que la chaîne gagnait en audience. Dès lors, celles correspondant à ces mots clefs ont été regroupées et comparées dans leurs approches.

Le caractère autoproduit des vidéos entraîne des particularités: le producteur, s'il semble à première vue être seul juge et modérateur de ses productions, dépend de son public (et donc des goûts de celui-ci) pour maintenir une visibilité et une audience. De plus, les vidéos ne s'inscrivent pas dans une dynamique de chaîne à proprement parler, dans la mesure où seule l'identité du Youtubeur et son rapport à l'islam font lien entre les sujets abordés. En effet, il est rare de trouver des cohérences quant à la continuité des vidéos entre elles, tant au niveau des sujets abordés que de la manière de

\footnotetext{
17 <https://www.youtube.com/channel/UCEVp9oosQo9HPVf7ldKmD4w>, dernière consultation le 14 mars 2014

18 <https://www.youtube.com/user/WestDawnMedia>, dernière consultation le 14 mars 2014.

${ }^{19}$ Nous choisissons ici d'utiliser la notion anglophone, qui reste à l'heure actuelle plus utilisée que sa traduction francophone « visionneur ».
}

tic\&société - 9 (1-2), 2015 
De la mise en scène pudique

comme expression d'une morale musulmane sur Youtube

les amener, ainsi que des repères disponibles (comme un générique désignant une catégorie précise de production). Néanmoins, toutes répondent à une volonté personnelle de partager une production ${ }^{20}$.

C'est ici la construction de la pudeur qui va nous intéresser, tout d'abord en tant que code moral du Youtubeur, mais aussi en tant que valeur à promouvoir et enfin en tant que normalisation de la relation aux autres, pour l'audience des chaînes.

\section{Analyse}

\subsection{Mise en scène de soi, entre fiction et vie quotidienne}

A priori, une distinction semble s'imposer entre les chaînes étudiées quant à leur alignement sur la vie quotidienne. Certaines productions relèvent officiellement d'une mise en scène narrative et se dénomment en tant que telles : «Muslim Short Movie $"{ }^{21}$ chez West Dawn Media et Islamicevent. D'autres semblent se rapprocher davantage de situations quotidiennes (productions d'Amenakin et Lebo2196) puisque le Youtubeur se place face à la caméra pour énoncer son avis sur une situation ou délivrer un conseil. En réalité, ces deux catégories sont moins faciles à discerner : en effet, chez Islamicevent les histoires proposées sont clairement fictionnelles (Rabi, dans «We Only Live Twice » revit, et dans "Dear Mum », se fait renverser et finit paralysé).

\footnotetext{
${ }^{20}$ Cette idée se retrouve dans le concept de communication personnelle de masse proposé par Bernard Miège (2008).

21 «Court-métrage musulman ».
} 


\section{Lucie LE GUEN-FORMENTI}

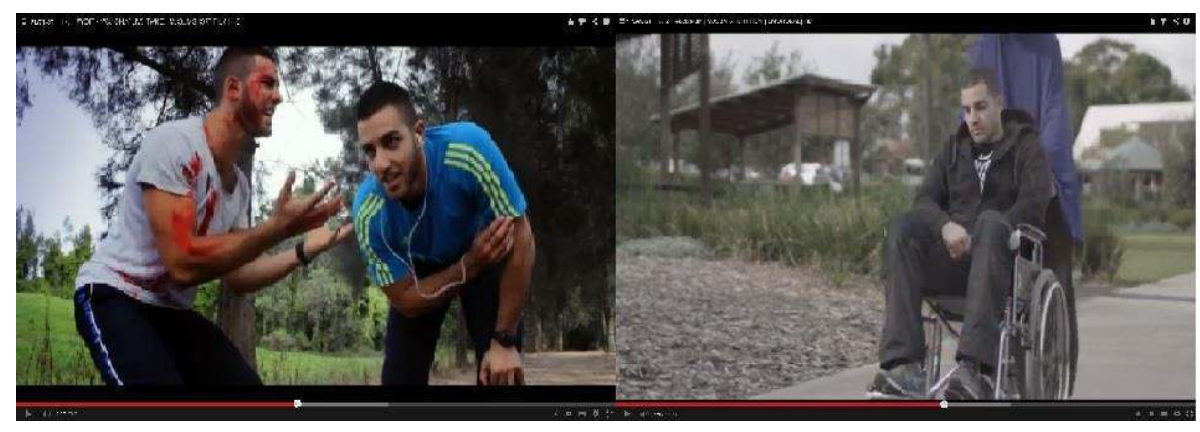

Figure 1 : captures d'écran Islamicevent, vidéos You Only Live Twice (à gauche) et Dear Mum (à droite).

Or le personnage principal n'est pas un acteur, mais un prêcheur musulman qui participe également à des vidéos sur l'islam ("Why You Will Love Islam », publiée par Lebo2196). C'est d'ailleurs par ces vidéos que l'on réalise que la voix off des court-métrages islamiques est la sienne. Ainsi, le lien entre implication personnelle et fiction est beaucoup plus ténu, de par cette unité positionnelle de l'émetteur et une grande similarité du message. La vidéo de Rabi (son vrai nom ou son nom complet n'est communiqué nulle part) réalisée par le groupe \#nohaters («Why You Will Love Islam») comprend aussi la participation d'un autre prêcheur, Kamal Saleh, que nous abordons dans cette étude pour ses slams et prêches.

tic\&société - 9 (1-2), 2015 
De la mise en scène pudique

comme expression d'une morale musulmane sur Youtube

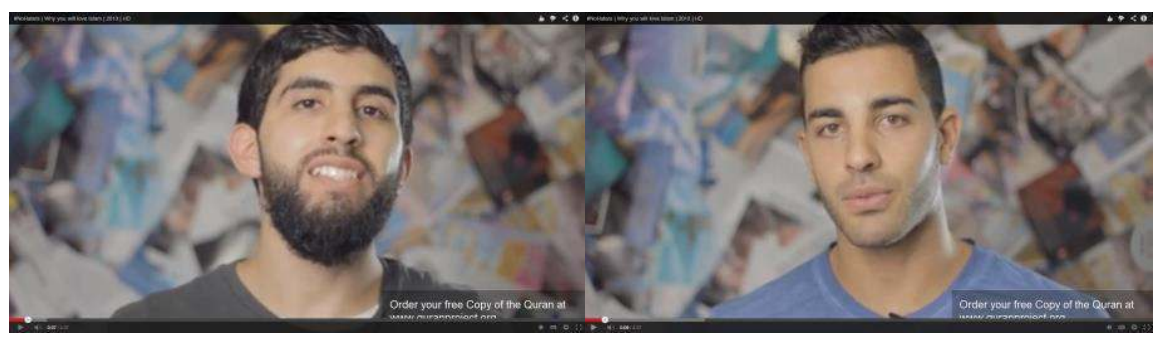

Figure 2 : capture d'écran Lebo2196, vidéo Why You Will Love Islam, intervention de "Rabi" (à gauche) et de « Kamal Saleh » (à droite).

Or, Kamal ne se présente pas lui-même en tant que tel : sa chaîne Youtube apparaît sous le nom de Lebo2196, et, si la paternité de ses vidéos est revendiquée (l'utilisation du « je » comme producteur de la vidéo «Islam is not a Religion of Peace - Muslim Response »), à aucun moment son nom n'est mentionné, durant la période au cours de laquelle nous effectuons notre recherche ${ }^{22}$.

22 II sera en revanche nommé ultérieurement par d'autres. 


\section{Lucie LE GUEN-FORMENTI}

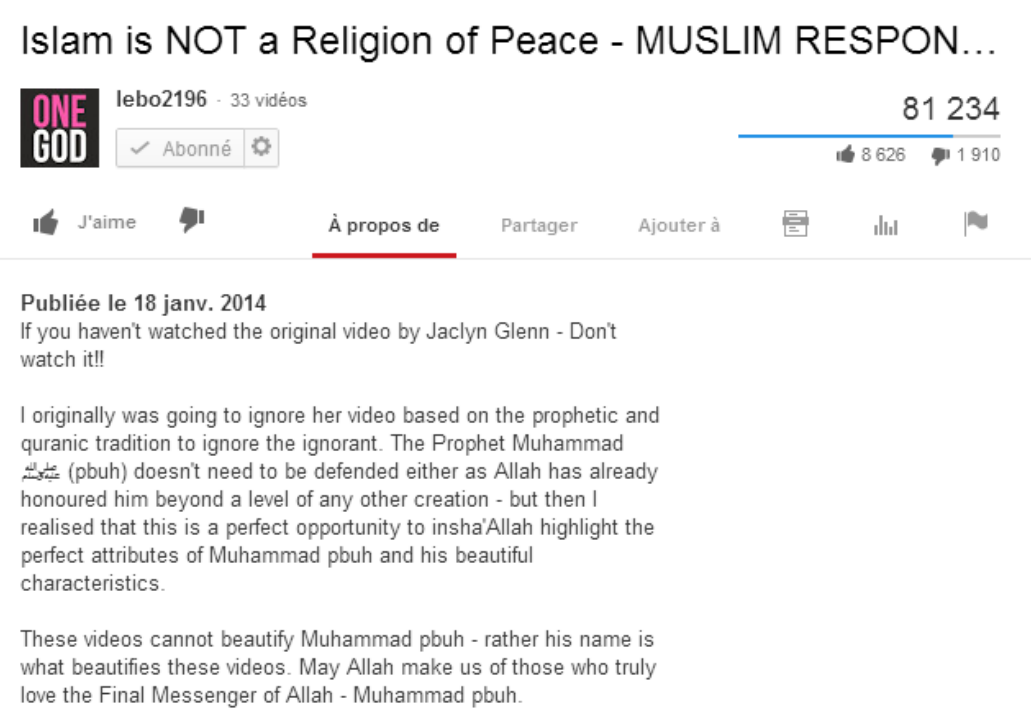

Figure 3 : capture d'écran du descriptif de la vidéo "Islam is Not a Religion of Peace - Muslim Response" de Lebo 2196.

Ce Youtubeur n'a pas non plus de page Facebook professionnelle sur laquelle ses abonnés pourraient suivre ses actualités et son nom n'apparaît d'ailleurs que lors des recherches quant aux polémiques de ses vidéos sur Google. Ce n'est donc pas Kamal qui est mis en avant comme producteur de ces vidéos, mais le personnage social qu'est Lebo2196.

L'aspect pudique se manifeste pour ces deux Youtubeurs par l'absence de référence à leur vie hors ligne, et le cloisonnement entre leur travail de Youtubeur et leur vie privée. De cette manière, parler de sujets privés semble cohérent et pudique, puisque c'est la dimension didactique et distanciée qui est mise en avant.

tic\&société - 9 (1-2), 2015 
De la mise en scène pudique

comme expression d'une morale musulmane sur Youtube

Le cas d'Amenakin est lui aussi ambigu. Si dans un premier temps, elle peut paraître être la plus conforme au genre du vlog ${ }^{23}$, présupposé comme relevant du champ de la vie quotidienne, elle va aussi participer à des vidéos bien plus fictionnelles, entraînant une narration.

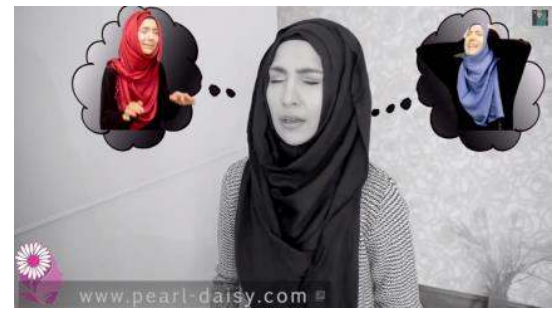

Figure 4 : capture d'écran, Amenakin "How to Not Touch Men".

Également, ses vidéos relevant du genre vlog ou tutorie ${ }^{24}$, disposent d'effets de post-production s'améliorant avec le temps: des scènes sont coupées pour éviter de montrer ses enfants, elle met en place une voix off sur ses tutoriels, ajoute des musiques, créant ainsi un décalage avec la vie hors ligne.

Enfin, West Dawn Media semble à première vue relever totalement du champ de la fiction. Or, sa principale production est un documentaire ("Napoleon, Life of an Outlaw»), et c'est de ce genre qu'elle va s'inspirer pour ses "short movies". Les court-métrages sont réalistes, et n'impliquent pas d'éléments irréels (contrairement à la présence du fantôme de Rabi dans "You Only Live Twice "); les personnages font face à des situations plausibles pour des musulmans vivant en occident, et recherchent des solutions applicables au monde tel qu'il est connu par l'audience supposée. De plus, les thématiques abordées (la recherche de l'âme sœur, les tensions professionnelles genrées, la crise d'adolescence, la séparation du couple...) sont les mêmes que celles d'Amenakin et Lebo2196, jugés a priori conformes à la vie quotidienne.

\footnotetext{
${ }^{23}$ Définition proposée par L'internaute : contenu à vocation de carnet, journal ou agenda réalisé sur support vidéo.

${ }^{24}$ Production à vocation didactique, concernant un logiciel ou une pratique culturelle.
} 


\section{Lucie LE GUEN-FORMENTI}

Ainsi, le rapport à la vie quotidienne n'est pas déterminant du «dévoilement » du Youtubeur. Celui-ci aménage des stratégies, à la fois pour se rapprocher de son public et pour s'en éloigner, afin de conserver une certaine distance pudique.

\subsection{La pudeur, une réciprocité}

Les vidéos étudiées revendiquent un rapport d'échange avec leur public ; non seulement dû à leur nature (prêches, vidéos morales, tutoriels) mais également par leur rapport à la pudeur qui implique une reconnaissance par autrui.

\subsubsection{La pudeur comme lien au public}

II est dès lors intéressant de voir que cette pudeur permet non seulement d'introduire ce lien sur une base didactique, mais également de créer une communauté autour d'une valeur morale, à visée plus large que la seule religion musulmane. Amenakin ne fait a priori que peu de distinction directe entre ses viewers musulmans et non musulmans. Elle attend d'eux une sympathie pour les causes qu'elle défend, et par ce biais, fait des vidéos pour expliquer les bases de la religion, tels que le jeûne, le vocabulaire, ou la modestie. En réalité, c'est cette dernière question qui est centrale pour elle, quelle que soit la religion : elle fait notamment une vidéo concernant les femmes voilées non musulmanes, présentant son soutien à ces jeunes femmes («Non-Muslim Hijabies »). C'est non pas un sentiment religieux qui fait lien, mais des valeurs communes.

Néanmoins, une sélection du public plus stricte s'opère dès 2011, puisqu'elle commence ses vidéos par " a-salam aleykum ladies ${ }^{25}$ («Drape Scarf Tutorial : Eva Green Inspired!»). La

\footnotetext{
25 «Que la paix soit sur vous mesdemoiselles!».
}

tic\&société - 9 (1-2), 2015 
De la mise en scène pudique

comme expression d'une morale musulmane sur Youtube

séparation, implicite jusqu'alors, est formalisée entre les genres, rendant cet espace exclusivement féminin. Un certain jeu va s'installer pour les Youtubeurs afin de construire un espace viable à cette ouverture sur le soi : Amenakin instaure un rapport privilégié avec ses viewers, une production médiatique thématique ${ }^{26}$, afin de justifier cette interpénétration de son travail de commerciale sur le web et de sa vie privée. En s'adressant uniquement à des femmes, elle délimite son public, crée un espace réservé à celles-ci. Bien qu'uniquement théorique puisque des hommes peuvent consulter ses vidéos, son propos est néanmoins justifié dans la mesure où elle ne s'adresse pas à eux, et qu'ils ne sont pas convié à ces conversations. Elle exprime ainsi une intentionnalité qui se positionne comme pure et bien fondée. Néanmoins, des nuances sont à soulever, dans la mesure où Amena n'apparaît jamais dévoilée. L'islam l'autoriserait pourtant à ôter son voile dans un espace féminin, mais elle va maintenir un cache sur ses cheveux, rappelant ainsi la limite de son positionnement genré.

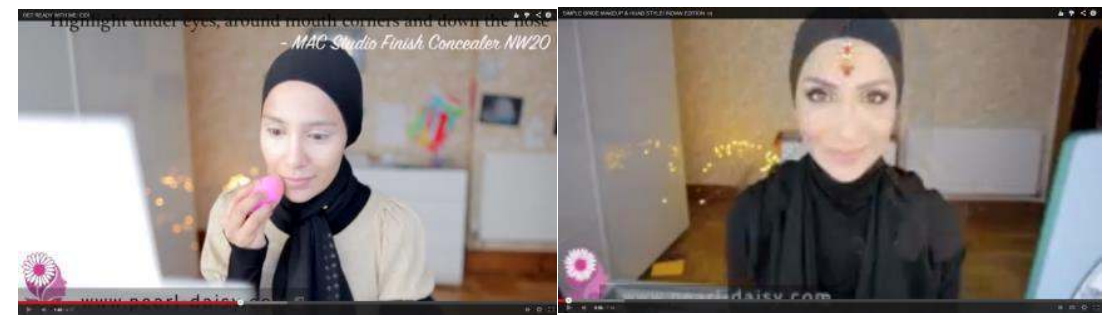

Figure 5 : capture d'écran Amenakin, vidéos "Get Ready With Me" (à gauche) et "Simple Bride Make Up \& Hijab Style! Indian Edition" (à droite).

Cette manière de se couvrir a en effet la charge symbolique du hijab (Al-Qasimi, 2010) par sa fonction métonymique. En 2014, un changement s'opère quand Osama, son mari, prend

${ }^{26}$ Contrat d'intercompréhension mutuelle et exclusive entre le producteur et les récepteurs, ainsi que promesse de pertinence (Maingueneau, 2013). 


\section{Lucie LE GUEN-FORMENTI}

la caméra et présente des vidéos. II instaure un "a-salam aleykum brothers and sisters », qui inclue cette fois les hommes, et présente la vidéo comme s'adressant à une communauté non plus unie autour du genre, mais autour de la $\mathrm{foi}^{27}$. Une seconde nuance à l'idée d'ouverture pluri-religieuse doit être également effectuée, dans la mesure où cette homogénéisation du public n'est que partielle: lorsqu'elle organise des jeux (vidéos "give-away»), les participantes doivent énoncer des réponses relatives à la foi. Les participantes non musulmanes sont ainsi exclues.

Les mini-films musulmans, s'ils semblent destinés à un public suivant l'islam, visent eux aussi un public hétéroclite : en abordant des situations problématiques pour la foi que leurs héros peinent à résoudre, les réalisateurs impliquent que leurs viewers ne sont pas uniquement de parfaits musulmans. Le couple, la fierté, la relation aux parents et l'exercice de la foi, sont autant d'éléments que le viewer doit remettre en question à propos de lui-même, en s'inspirant des vidéos pour résoudre ces problèmes. La pudeur est donc présentée comme une clef d'insertion et de réussite sociale. Les vidéos étudiées ne présupposent pas d'un haut niveau de religiosité de leur audience. Dans cette mesure, elles vont, en parallèle de leur discours, développer une dimension didactique visant à améliorer le musulman à qui elles s'adressent. L'audience estelle humble? Applique-t-elle l'amour filial ? Sait-elle pardonner?

Ainsi, la distinction du public via le spectre de la pudeur est floue: les séparations qu'elle implique semblent rester volontairement vagues.

\footnotetext{
${ }^{27}$ L'appellation arabe pour communauté est « umma », en référence à la mère. Dans un même ordre d'idée, les membres de la communauté vont référer aux autres comme "frères" et "sœurs". L'utilisation de la tournure idiomatique arabe traditionnelle et des termes d'adresse usités entre musulmans tend donc à proposer une séparation religieuse plutôt que genrée.
}

tic\&société - 9 (1-2), 2015 
De la mise en scène pudique

comme expression d'une morale musulmane sur Youtube

\section{jugement \\ 3.2.2. La pudeur comme élément pédagogique de}

La pudeur peut aussi être un élément d'inclusion et d'exclusion partagé par la communauté (Tillion, 1966). En tant que jugement de la morale, elle implique une évaluation par le public du comportement du Youtubeur, ainsi que des autres followers.

En ce sens, l'idée de Mu'aththir (Hirschkind, 2012) se retrouve partiellement dans les vidéos Youtube étudiées : les Youtubeurs vont instaurer un lien particulier avec le public, l'intégrer à leur production. Kamal va s'adresser à eux, West Dawn Media (dans "The Hijab ») et Amena vont régulièrement demander l'avis du public sur les productions pour savoir si tel ou tel sujet doit être continué, si la forme convient. Dans cette mesure, le public est postulé comme une entité, qui doit prononcer un avis, relayé par les commentaires, les likes et les vues.

Cette manière de voir le public comme une communauté, induit une vision particulière reproduisant les rapports de mosquée: les Youtubeurs postulent un sentiment d'appartenance relayé par les incitations à s'abonner à la chaîne, à la page Facebook, où dans le cas d'Islamicevent et Amena, participer aux évènements organisés.

Dans une certaine mesure, une régulation morale va s'instaurer entre les participants à la conversation. Comme dans le cas des khutbas en ligne (Hirschkind, 2012), un code moral implicite sous-tend ce que peuvent énoncer les participants: dans la vidéo "Get Ready with me, Eid" d'Amena, les commentaires jugés déplacés d'un viewer sont d'emblée critiqués, bien qu'ils n'aient pas été supprimés par la Youtubeuse elle-même. 


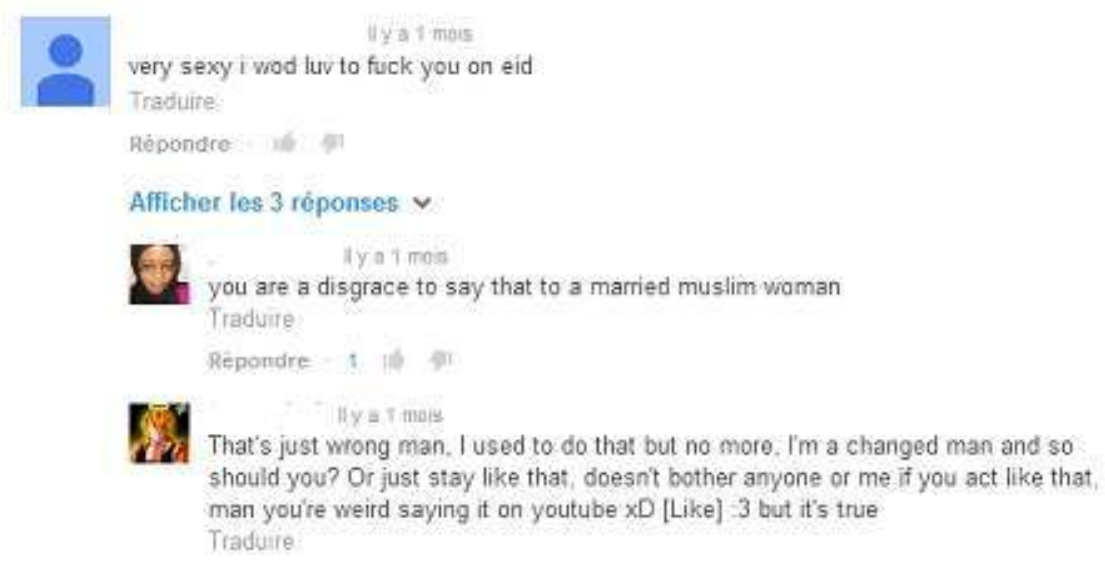

Figure 6 : capture d'écran de commentaires pour la vidéo d'Amenakin "Get Ready With Me : Eid".

Or, dans une vidéo réalisée en commun avec Potholer54 ${ }^{28}$, la productrice énonce qu'elle effectue un tri préalable à la publication des commentaires, supprimant ceux qui sont manifestement racistes et injurieux. Dès lors, il est intéressant de voir que les commentateurs ne correspondant pas aux attentes des autres viewers vont se voir rappeler à l'ordre. En ce sens, les viewers respectent majoritairement l'idée d'un contrat moral établi entre le Youtubeur et eux : ils vont tenter de proposer une image d'eux-mêmes en accord avec les valeurs enseignées par le Youtubeur.

Dans le cas d'Islamicevent et de Lebo2196, l'influence de l'avis des viewers est également claire : si les vidéos étudiées ne représentent qu'une minorité de leur production, ce sont celles qui provoquent le plus de vues, et qui vont motiver les productions les plus récentes. Les prêches d'imams sont remplacés par des productions plus personnelles, sujet de réel engouement. Dans une certaine mesure, il semble que ce soit les personnalités de ces Youtubeurs qui intéressent le public, leurs avis personnels. En effet, si, jusqu'alors, les publications par Rabi de vidéos de prêcheurs et d'évènements lui accordaient du crédit ${ }^{29}$, il se protégeait derrière la parole d'un

\footnotetext{
$28<$ https://www.youtube.com/watch?v=Gu-BuHPXB0s> (dernière consultation le 14 mars 2014), suite à leur altercation concernant les droits Youtube et la théorie de l'évolution.

${ }^{29}$ Proposer des vidéos sur sa page revient en quelque sorte à citer la personne, en exprimant un accord avec son discours (Maingueneau, 2013).
}

tic\&société - 9 (1-2), 2015 
De la mise en scène pudique

comme expression d'une morale musulmane sur Youtube

collectif : la ummah. Par la suite, il va se mettre en scène personnellement, sous couvert d'un contexte de fiction, et donc se dévoiler en proposant une vision personnelle de la responsabilité du musulman vis-à-vis de sa religion.

Si une même analyse peut être proposée à propos du cas de Kamal Saleh, la transition est encore plus aboutie. Le succès massif de ses premiers slams va le pousser à produire régulièrement des prêches et des Spoken Words, plutôt consensuels: le message proposé n'est en rien novateur et correspond à des valeurs très généralistes. Néanmoins, sa personnalité se dégage petit à petit : dans les premières vidéos, il se présente devant un fond gris et par la suite, va commencer à se mettre en scène dans des situations auxquelles le public peut s'identifier: dans "Muslims vs_Atheists Response \#jihadagainstignorance ", il se présente devant son ordinateur, et dans "Islam is not a Religion of Peace-Muslim Response ", on peut le voir dans son lit en pyjama, en train de lire un message sur son téléphone. 


\section{Lucie LE GUEN-FORMENTI}

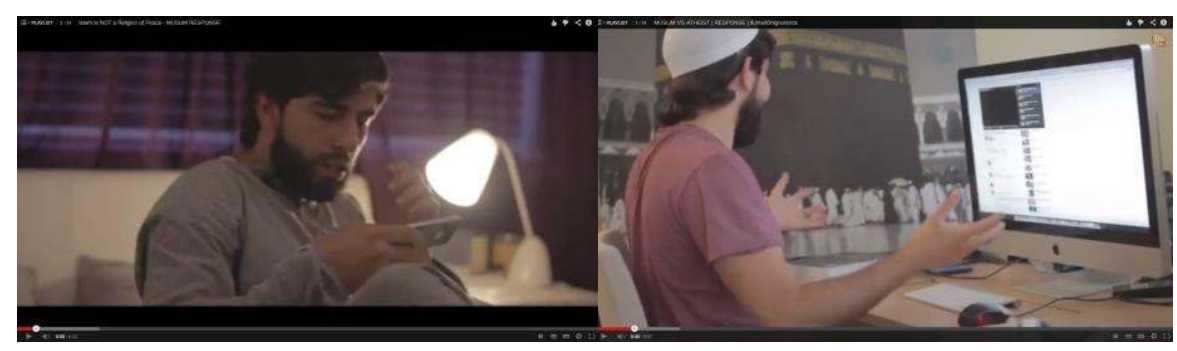

Figure 7 : captures d'écran Lebo2196 "Why Islam is NOT a Religion of PeaceMuslim Response" (à gauche) et "Muslim VS Atheist » (à droite).

Cette proximité du Youtubeur est également présente chez Amenakin. Au fur et à mesure des vidéos, on peut voir une imbrication de sa vie privée à sa vie publique de Youtubeuse : dans les premières, elle se présente seule. Souvent, on peut entendre les personnes qui l'entourent hors champ, mais elles n'apparaissent jamais en ligne. Face à l'engouement de ses followers pour sa personnalité, elle va peu à peu intégrer ses sœurs, son mari et récemment son père à ses vidéos.

tic\&société - 9 (1-2), 2015 
De la mise en scène pudique

comme expression d'une morale musulmane sur Youtube

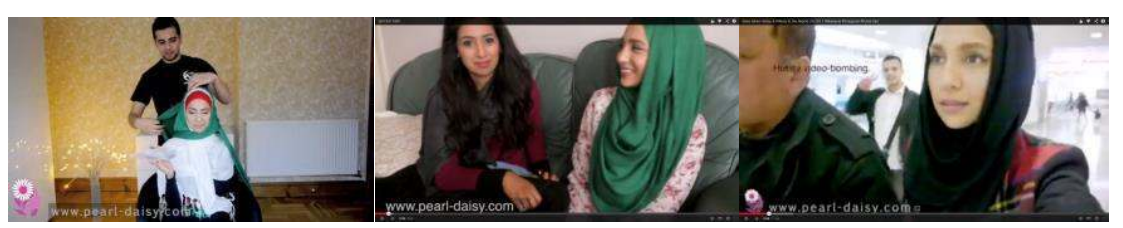

Figure 8 : Captures d'écran Amenakin "My Husband Do my Hijab" (à gauche, avec son mari), "Sister Tag" (au centre, avec sa sœur), "Malaysian Vlog, Getting to the Airport" (à droite, avec son père).

Régulièrement, elle produit des vidéos pour répondre à des questions personnelles ${ }^{30}$, organise des rendez-vous avec ses fans et se voit obligée de faire une vidéo ("Why We don't Show our Children on Youtube ") face aux demandes nombreuses et récurrentes des fans a propos de ses enfants. Cette dernière réglementation de la pudeur implique une distanciation de l'émetteur vis-à-vis de certains pans de sa vie privée.

Se dénote ainsi une certaine exemplarité du Youtubeur : ce n'est pas uniquement le message religieux qui importe, mais également sa personnalité. Le public cherche à se sentir proche des individus, à intégrer leur quotidien.

\subsection{Le diktat d'un message pudique}

Cet intérêt du public pour l'individu youtubeur implique néanmoins un questionnement quant aux attentes des viewers : dans la mesure où la survie de la chaîne dépend de son audience, ses préférences peuvent impliquer une manière particulière de se présenter en ligne et une logique médiatique.

\footnotetext{
30 Plus que marquée par une perspective exhibitionniste, cette démarche s'inscrit dans une tradition d'exemplarité en islam (Kreil, 2010).
} 


\section{Lucie LE GUEN-FORMENTI}

\subsubsection{L'influence du récepteur}

Cette demande du public est ambigüe, et, en ce sens, peut être rapprochée de l'analyse que donne François Jost (2007) de la téléréalité : certaines vidéos terre-à-terre ne rencontrent que peu de succès («Cabbie Attack» pour West Dawn Media, ou les revues de produits bébé pour Amenakin). Le viewer n'est pas réellement intéressé par une vision «paparazziesque », si l'on peut dire, du Youtubeur ; s'il désire le connaître, obtenir des preuves de sa valeur en tant que musulman, c'est néanmoins une vision rêvée de cette vie qui est valorisée. On peut alléguer que le public n'est pas à la recherche d'une réalité prosaïque, mais d'un semblant de réalisme. Par contre, les productions relevant d'un certain champ romantique et orientaliste sont sujettes à un plus grand succès. Le viewer va plaquer un certain nombre de stéréotypes (une recherche de l'exotisme, de l'amour, du voyage et du rêve) sur les attentes qu'il peut avoir envers le Youtubeur, et orienter ses visionnages en fonction. L'exemple le plus frappant est celui des vidéos d'Amena : lors de son voyage en Inde avec son mari (qui est retranscrit à travers 4 vidéos), le vlog suscitant le plus de vues est celui intitulé "Wedding Vlog ». De la même manière, ses vidéos en rapport avec les thématiques du mariage ("Get Ready with Me, Indian Wedding Edition ", "Wedding Hoojab Tutorial \& OOTD », «Hijaab Tutorial, Elegant Wedding Gest »...) obtiennent généralement plus de 100000 vues. En ce sens, nous pouvons parler de personnage, plus que de personnalité : les Youtubeurs doivent lisser leurs caractères et leur vie profane afin de correspondre à ces attentes.

Ce diktat indirect des consommateurs de la chaîne semble perçu par les Youtubeurs, qui vont parfois adapter leur discours en fonction. Amena notamment, produit la vidéo « I'm a Girl on Youtube » en réponse à une vidéo parodique de Benjamin 


\section{De la mise en scène pudique \\ comme expression d'une morale musulmane sur Youtube}

$\mathrm{Cook}^{31}$ qui définit ce que doit faire une Youtubeuse. Même si elle va critiquer le point de vue que propose Benjamin sur la manière que peut avoir une femme d'accéder à la reconnaissance via Youtube («Beauty guru vlogger») 32, les vidéos qui la font connaître sont celles se conformant à ce stéréotype. Ses vidéos proposant un fond réflexif et critique sont par ailleurs beaucoup moins visionnées ("Sheikh Google "33, par exemple, ne suscite que 62000 vues tandis que ses vidéos dépassant 500000 vues sont toutes des tutoriels de mode). Finalement ce sont les vidéos concernant les problématiques relatives à la gestion de la vie privée qui vont intéresser.

\subsubsection{Le couple et l'amour, des thématiques privilégiées}

Si le couple et l'amour sont a priori des thématiques relevant en partie de la sphère privée ${ }^{34}$, ce sont celles qui vont provoquer le plus d'intérêt, et donc susciter des productions. Dès lors, il est intéressant de porter le regard sur la manière que vont avoir les Youtubeurs de présenter ces éléments de manière pudique.

Le point de vue sur le couple, d'une manière générale, est plutôt similaire. Seul Islamicevent présente cette relation comme étant source de perversion : métaphoriquement, dans "You Only Live Twice », Rabi décède suite au peu d'attention qu'il accorde à sa foi, en comparaison de sa vie profane (il privilégie la conversation téléphonique avec son amie plutôt que la voie tracée...). En revanche, les autres Youtubeurs l'envisagent d'un point de vue moins dramatique, et créent des

\footnotetext{
${ }^{31}$ Chaîne Nine Brass Monkey, <https://www.youtube.com/user/ninebrassmonkeys>, dernière consultation le 14 mars 2014.

32 "Vlogueuse et gourou beauté », soit quelqu'un qui propose des vidéos de mode et de maquillage, ou traite uniquement de thématiques relatives à l'apparence.

${ }^{33}$ Vidéo commune à plusieurs Youtubeurs critiquant les dérives d'internet quant à l'auto-institution de "gourous musulmans ».

${ }^{34}$ Phénomène déjà remarqué par Bernard Miège (2008) lorsqu'il indique l'intérêt des médias de masse pour la « privacy » (thématiques privées).
} 


\section{Lucie LE GUEN-FORMENTI}

vidéos exclusivement centrées sur les difficultés rencontrées dans le couple.

Pour Kamal, la lutte se situe avant la mise en couple réelle (« Love, Marriage and Fairytales ») « rather search to find Allah first, and once you find him, you're garanteed to find her $\gg 35$. C'est donc par la remise en question, la foi et la relation à Dieu que le couple peut se construire. Cette idée se retrouve également chez West Dawn Media, mais proposée de manière différente : le couple y est une construction difficile, notamment concernant la vie commune. Dans «Change of Heart», le couple connaît une crise due à la vie passée en tant que non pratiquante de la protagoniste. C'est l'acceptation de la remise en cause, et le retour vers Dieu qui permettent la pérennité du couple, malgré des écarts antérieurs faits à la religion. La vidéo prône l'idée d'une perfection seulement divine, et une nécessité d'accepter l'erreur humaine :

«- I looked in you as my dream girl [...]

- I'm flattered you treated me so highy but it's unfair, I'm a human being [...]

-l know »36

Cette idée de l'acceptation de l'humanité de l'autre se retrouve d'ailleurs chez Amenakin, à travers les nombreuses vidéos qu'elle poste sur le couple et sur la recherche de l'âme sœur. Malgré l'aspect réaliste qu'elle met en avant (ne pas avoir trop d'illusions ni d'attentes) et les valeurs morales qu'elle promeut, elle rejoint néanmoins une vision idéalisée du couple telle que décrite par Kamal Saleh: les vidéos taggées ${ }^{37}$ «Osamena », la mettant en scène avec son mari, proposent la

\footnotetext{
35 «Recherche plutôt Dieu en premier, et une fois que tu l'auras trouvé, tu seras assuré de la trouver elle ».

${ }^{36}$ « Je te voyais comme la fille de mes rêves [...] / Je suis flattée que tu me tiennes en si haute estime, mais c'est injuste, je suis juste humaine [...]. / Je sais bien. "

${ }^{37}$ Marquées du \# (hashtag) afin d'être relayées sur Twitter ou dans les moteurs de recherche.
}

tic\&société - 9 (1-2), 2015 
De la mise en scène pudique

comme expression d'une morale musulmane sur Youtube

vision d'un couple parfait et heureux et ne questionnent à aucun moment la possible difficulté d'une vie commune.

Ainsi, certains éléments à première vue privés se voient recouverts d'un habillage socialement accepté, respectant les règles de pudeur : il est dans cette mesure correct d'aborder la construction du couple d'un point de vue d'acteur social, tandis que l'amour sera un sujet tabou. La pudeur va s'instaurer en tant que valeur positive dans le jugement de l'individu (Tillion, 1966) : les Youtubeurs, s'ils veulent être crédibles et suivis dans leur discours religieux, doivent faire preuve d'un certain jeu de valeurs morales, dont le tandem honneur/pudeur. Doit donc se développer une certaine monstration de cette pudeur, afin d'appuyer le discours.

\section{Conclusion}

Tout au long de cette étude, le questionnement a porté sur la motivation de ces vidéos, et le rôle social qu'elles pouvaient avoir. Avait été postulé leur rôle interstitiel là où les infrastructures religieuses et associatives ne parviennent pas à répondre à la demande d'une population musulmane requestionnant tant ses pratiques que son rapport à la foi. Dès lors, le questionnement avait été de savoir quelles ressources mobilisent ces vidéos pour répondre à ces attentes, tout en respectant les normes religieuses traditionnelles. Si une telle monstration de la pudeur semblait difficilement réalisable d'un point de vue religieux, il apparaît que non seulement cette pudeur est l'élément qui permet de valoriser et de juger le discours tenu et la personne qui le tient, qu'elle guide un certain code référentiel afin de porter un regard sur le monde et le comportement social, mais également qu'une certaine forme de pudeur particulière se développe. En un sens, cette affiliation à la pudeur, qu'on pourrait qualifier de métonymique, va récupérer des attributs définis et les plaquer sur une réalité autre que le contexte usuel, et avec une visée différente. En découle l'idée de "dévoilement transgenre », puisque s'opère cette idée de démasquer une partie de la personnalité, quel que soit le sexe, et quel que soit le type de vidéo. Les attributs sont identifiés comme relevant d'un code moral pudique (ou de la 


\section{Lucie LE GUEN-FORMENTI}

modestie, ou de l'honneur, jugées être des valeurs connexes régissant de la même manière les rapports sociaux), les comportements auxquels ils s'associent revêtent la valeur qu'ils véhiculent, permettant ainsi l'apparition de nouvelles pratiques et de nouveaux jugements en rapport avec ces pratiques, mais s'inscrivant dans une dynamique traditionnelle. Le Youtubeur répond à une demande, une volonté de mimétisme fondé à la fois sur une déliquescence du lien communautaire ${ }^{38}$ mais aussi sur une tradition fortement tournée vers l'exemplarité. Se dégage un nouvel individu social, qui, à première vue, aurait pu paraître impudique et relever de la sphère privée, mais qui va pourtant subir une codification stricte, tant de la part de l'émetteur que des récepteurs.

\section{Références bibliographiques}

AL-QASIMI N., 2010, «Immodest Modesty : Accommodating Dissent and the "Abaya-as-Fashion in the Arab Gulf States ", Journal of Middle East Women's Studies, vol.6, $\mathrm{n}{ }^{\circ 1}$, pp.46-74.

BOUHDIBA A., 1984, «La société maghrébine face à la question sexuelle ", Cahier internationaux de sociologie, vol.76, pp.91-110.

CARDON D., 2013, «Du lien au like sur internet, deux mesures de la réputation », Communications, vol.2, $\mathrm{n}^{\circ} 93$, pp.173-186.

CARDON D., 2008, «Présentation », Réseaux, vol.6, n¹52, pp.7-17.

CESARI J., 2004, I'Islam à l'épreuve de l'occident, Paris, La Découverte.

\footnotetext{
${ }^{38}$ Pour Olivier Roy (2004), inhérent à la vie en occident.
}

tic\&société - 9 (1-2), 2015 
De la mise en scène pudique

comme expression d'une morale musulmane sur Youtube

Coran [1967], traduction D. Masson, Paris, Gallimard.

DENOUËL J., 2011, «Identité», Communications, n88, pp.75-82.

DUPONT V. et B. TILLIER, 2012 «Présentation », Sociétés \& représentations, vol.1, $\mathrm{n}$ \%3, pp.7-10.

DUPRET B., E. KLAUS et Z. GHAZZAL, 2010, « Commenter l'actualité sur internet: la structure d'intelligibilité d'un forum de discussion arabe », Réseaux, vol.2-3, n¹60-161, pp.286-317.

GEORGES F., 2011, "L'identité numérique sous emprise culturelle, De l'expression de soi à sa standardisation ", Les cahiers du numérique, vol.11, $\mathrm{n}^{\circ} 1, \mathrm{pp} .31-48$.

HAENNI P., 2005, L'islam de marché, Paris, Le Seuil.

HIRSCHKIND C., 2012, "Experiment in devolution on line: the Youtube khutba", Middle East studies, n ${ }^{\circ 44}$, pp.5-21.

HUGON S., 2011, “Communauté », Communications, n88, pp.37-45.

JOST F., 2007, Introduction à l'analyse de la télévision, Paris, Ellipses.

KHOSROKHAVAR F., 2003, Les nouveaux martyrs d'Allah, Paris, Flammarion.

KREIL A., 2010, "Se faire cheikh au Caire, Exemplarité et intériorité religieuse », Archives de sciences sociales des religions, $\mathrm{n}^{\circ} 149$, pp.255-272.

MAINGUENEAU D., 2013, Analyser les textes de communication, Paris, Armand Colin.

MIEGE B., 2008, «Médias, médiations et médiateurs, continuités et mutations ", Réseaux, vol.2, n¹48-149, pp.117146.

OSSMAN S., 2002, Three faces of beauty, Casablanca, Paris, Cairo, London, Duke University Press.

ROY O., 2004, L'Islam mondialisé, Paris, Le Seuil.

TILLION G., 1966, Le harem et les cousins, Paris, Le Seuil.

TISSERON S., 2011, "Intimité et extimité ", Communications, $n^{\circ} 88$, pp.83-91. 